\title{
Parallel Waveform Relaxation and Matrix Solution for Large PEEC Model Problems
}

\author{
Giulio Antonini* Jonas Ekman+ and Albert E. Ruehli§ \\ * Dept. Electrical Eng., Univ. of L'Aquila, 67040, L'Aquila, Italy (antonini@ing.univaq.it) \\ + LuleåUniversity of Technology, Luleå, Sweden (jonas.ekman@sm.luth.se) \\ §IBM T. J. Watson Research Center, Yorktown Heights, NY 10598, USA (ruehli@us.ibm.com) \\ (Authors are in alphabetic order)
}

\begin{abstract}
Excessive compute time is becoming a key problem for high performance system modeling as the complexity of the electromagnetic and circuit models is increasing. At the same time the PEEC models are locally becoming more complex with the increased importance of dielectric and skin-effect losses. In this paper, we consider a combined approach where waveform relaxation is used for the predominant weak coupling while a Gaussian matrix solver is used for the parallelization of the strongly coupled parts of the EM/Ckt solver.
\end{abstract}

\section{INTRODUCTION}

Excessive compute time is becoming a key problem for high performance system modeling as the complexity of the electromagnetic and circuit models is increasing. At the same time the local complexity of the models is increasing with the importance of dielectric and skin-effect losses. These loss models may consist of local circuits in the PEEC context. In this paper, we use the volume Partial Element Equivalent Circuit (PEEC) approach [1]. In the last 25 years, many waveform relaxation (WR) and WR-like concepts have been applied to SPICE type circuits by many researchers, e.g. [2], [3]. It was always well known that the best results with WR can be obtained with parallel processing. Hence, new parallel solution techniques are of fundamental importance for the solution of this problem. Today, multi-core chip machines are available with an increasing number of processors at a very affordable price. Algorithms which are suitable for a variety of different machines are becoming more important. While new automatic compilers for multi-core processors are being developed today, the performance can be greatly enhanced with application-specific algorithms. In this paper, we will show how we can take advantage of the specific properties of the EM/Ckt systems at hand. The only early work we are aware of on WR for EM problems is [4]. In recent work, we considered WR for the purpose of solving large EM/Ckt systems using parallel matrix techniques [5] and WR [6]. The WR approach has been utilized for a multitude of circuit and other applications, e.g. [7], [8], [9]. However, this may result in widely varying levels of performance due to the restriction for the parallel processing to weakly coupled SubSystems (SSy). This in turn limits the performance of the algorithms. Fortunately, PEEC models, like other integral equation based models, do involve a very large number of weak couplings. Unfortunately, one problem is that some of the SSy may form large, strongly coupled blocks. This can degrade the performance as will be clear from the explanations given below. To solve this problem, we suggest in the paper a combined approach based on [5] and [6] where WR is used for the predominant weak couplings while a parallel Gaussian matrix solver is used for the parallelization of the strongly coupled parts of the overall system. Hence, the challenge is to combine the approaches efficiently in such a way that the best overall performance can be achieved. The fundamental solution approach pursued in this paper is based on the following two observations:

Observation 1: (Sparse parallel Gauss matrix solver)

This approach does not have the usual convergence issue which iterative solvers have. As is shown in the left side of Fig. 1, their performance usually saturates with respect to the number of processors. This approach works best for parallel systems with a low latency.
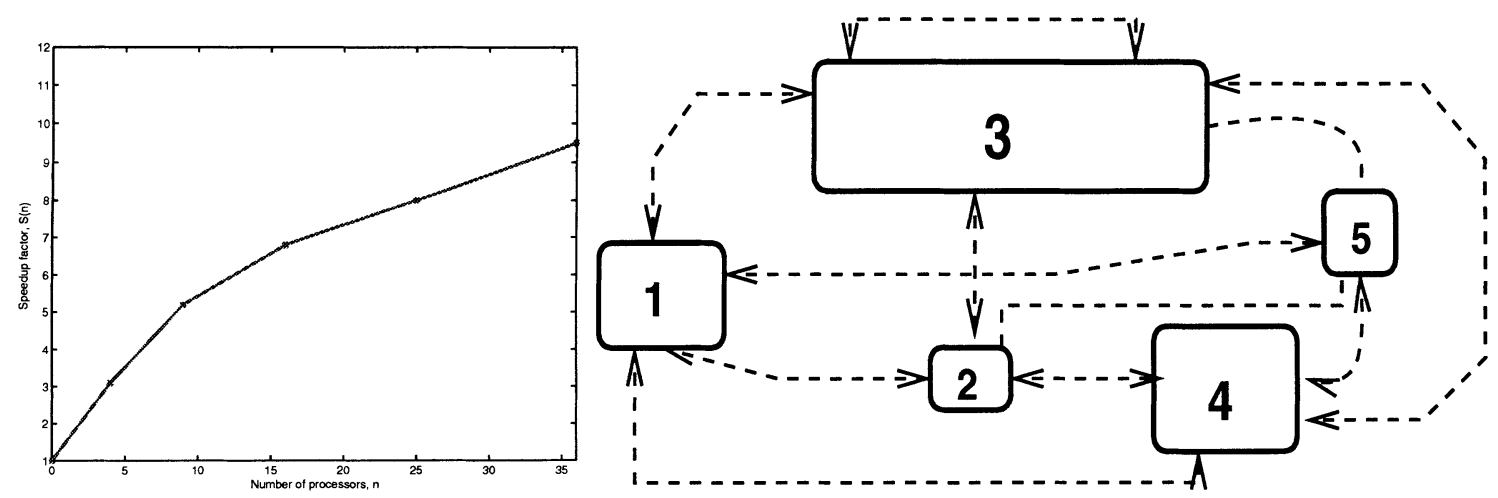

Fig. 1. Left: Example efficiency for matrix solver. Right: Example of a small system partitioned into decoupled SSys 
Observation 2: (Classical waveform relaxation)

The classical WR approach works best for weakly coupled system. In this case, convergence is very fast and it can be guaranteed. This approach is efficient for large systems with many subsystems and for a large number of time points involving partial or total time waveform since this results in an excellent processor to communication ratio. Hence, larger parallel system latency can be tolerated.

From this it is very clear that the two techniques are complementary in their utility and lead to excellent combined algorithms. The strategy of the WR approach used here - in contrast to other more conventional iterative techniques - is to split or partition the system at weakly coupled connections such that convergence can be accomplished in only a few interactions. Importantly, this guarantees convergence for all problems. Partitioning has to be done as a pre-processing step, an approach which has been utilized successfully in the past. Hence, the partitioning strategy is fixed beforehand while the number of WR iterations is determined by a convergence test which compares the maximum difference between two consecutive iterations. Today, the much higher frequencies in VLSI circuits and packaging lead to problems with models with a much larger number of mutual coupling elements. A very good example of this is the WR work for multiple transmission line coupling [9]. It was shown in that paper how the multiple inductive and capacitive couplings between the transmission lines are solved with the TR-WR algorithm used. Similarly, for PEEC we can identify all coupled partial inductances, capacitances and potential coefficients to find the ones which yield a small enough coupling coefficient for decoupling. Then, only the large couplings need to be evaluated non-iteratively while all other couplings can be handled with WR. Another source of potential partitioning for full wave (Lp,R,P, $\tau)$ PEEC models can be retardation or delay, since all the delayed values for the elements can be derived from the known past values in time. Each transient analysis on a processor consists of the solution of a system of the form (1). This corresponds to a block or $S S y$ shown in the example partitions Fig. 1 right. We subdivide the variables according to the ones in the self-system and the ones which couple to other systems in the form

$$
\begin{gathered}
\mathcal{C}^{*}{ }_{0} \dot{x}+\mathcal{G}^{*}{ }_{0} x=\sum_{i} \mathcal{G}^{*}{ }_{i} \boldsymbol{x}\left(\boldsymbol{t}-\tau_{\boldsymbol{i}}\right)+\sum_{i} \mathcal{C}^{*}{ }_{i} \dot{\boldsymbol{x}}\left(\boldsymbol{t}-\tau_{i}\right)+ \\
\sum_{i} \mathcal{B}^{*}{ }_{i} \boldsymbol{u}_{\boldsymbol{i}}\left(\boldsymbol{t}-\tau_{i}\right)+\sum_{i} \mathcal{C}^{+}{ }_{i} \dot{x}\left(t-\tau_{i}\right)+\sum_{i} \mathcal{B}^{+}{ }_{i} \boldsymbol{u}_{\boldsymbol{i}}\left(\boldsymbol{t}-\tau_{\boldsymbol{i}}\right)
\end{gathered}
$$

where the elements with an $*$ pertain to the self-subsystem and + represents the elements which couple to the other subsystems. All these variables are equipped with voltage waveforms which are updated each time a subsystem is scheduled and solved on one of the processors. Parallel processing has been used for speeding up the computation of the delayed past value computations for a time domain integral equation approach in [10]. For the (WR)PEEC approach presented in this paper, we subdivide the system into smaller subsystems so that tasks can be assigned to the processors in such a way that we can keep them simultaneously as busy as possible.

\section{Partitioning Strategy}

A WR algorithm consists of several key steps: the partitioning into $S S y$; the ordering to determine the order of the SSys to be dispatched; and, the scheduling of the SSy transient analysis on the different processors [3]. We will for efficiency reasons use the newly computed waveforms immediately once they become available with what we call Gauss-Seidel WR updating. The appropriate best ordering and scheduling of the SSy is an efficiency question. For this short paper, we concentrate on the important partitioning step by splitting the system into internally strongly coupled SubSystems $S S y$ so that they can be processed independently. Here we will consider only EM based systems where the externally connected elements are only simple basic circuit elements like sources and load resistances or capacitances. After the partitioning step the connections between the SSys will consist of a multitude of mostly weakly coupled elements. In general, we have to distinguish between four different situations: either strong and weak $d c$ couplings or strong and weak $a c$ couplings. As considered in [2], [3], we build $S S y$ s by connecting all strongly coupled elements into one $S S y$ coupled to other SSys by weak coupling elements. Hence, the size of the SSys will vary depending on the coupling situation. As will be discussed below, the parallel matrix solvers will be used to even out the compute time for the different size SSys.

The identification of the weak PEEC couplings is of key importance for the formation of the SSys. In earlier work, inductive couplings were not considered. However, it was shown in [9] that inductive couplings, as is shown in Fig.2 Left, can be partitioned efficiently. Due to the partitioning step only weak couplings or oneway couplings exist between two SSys. This is accomplished by making sure that interactions between the neighboring circuit variable $\boldsymbol{x}$ contract sufficiently in a forward and backward iteration with a neighboring SSy. Again, all interactions between a node with MNA current, voltage, charge or flux variables $\boldsymbol{x}$ and all connections to the exterally connected nodes in other $S S y$ s must be weak. The contraction factor $\gamma$ is found by considering the forward and backward mapping at an interface element where the local mapping is $x^{k+1}=\gamma_{\ell} x^{k-1}$. Since we want the WR convergence to be within a few iterations we need to have a relatively small contraction factor like $\gamma_{\ell}<0.4$. An important positive observation is that for the majority of the PEEC couplings we do have $\gamma<<0.1$. Hence, one or two iterations will suffice for a large portion of the couplings. A simple first 


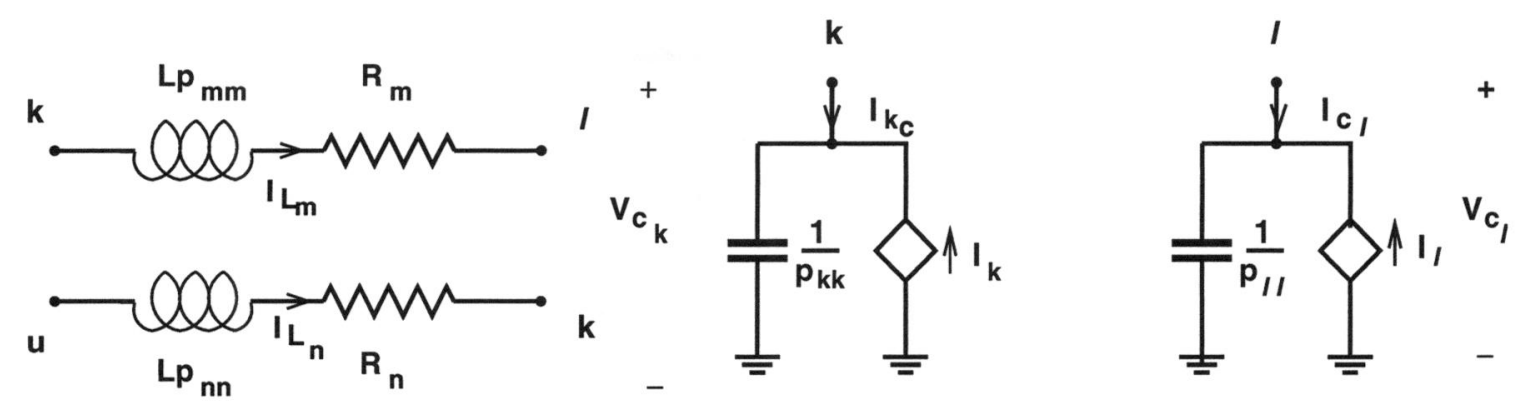

Fig. 2. Left: Simple inductive coupling between two PEEC cells. Right: Simple capacitive coupling between two PEEC cells
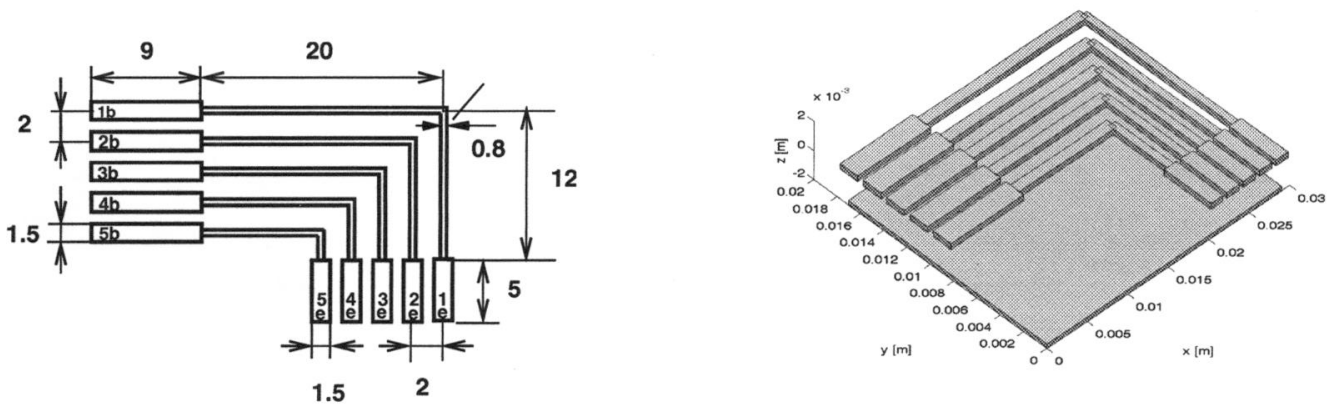

Fig. 3. Left: Connector plane measurements. Right: Connector plane with one ground sheet example

order estimation of the worst couplings can be made by considering the local circuit element couplings only. A formulation for the inductive couplings between two partial inductances in two different SSys as shown in the left side of Fig.2 is given by $\gamma_{\ell}=L p_{m n}^{2} /\left(L p_{m m} L p_{n n}\right)$. The capacitive coupling circuit between any two PEEC cells is shown in the right part of Fig. 2 with the coupling equations

$$
i_{c k}(t)=\frac{1}{p_{k k}} \frac{\partial \phi_{k}}{\partial t}-\sum_{n \neq k} \frac{p_{k n}}{p_{k k}} i_{c n}\left(t_{k n}^{\prime}\right)
$$

where $i_{c k}$ is the total capacitive current for cell $k$ and the retardation time is $t_{k n}^{\prime}=t-\frac{R_{k n}}{c}=t-\tau$ where $R_{k n}$ is the distance between cells $k$ and $n$ and $c$ is the speed of light. The coupling factor of this case can be computed as $\gamma=\left(p_{k n} p_{n k}\right)\left(p_{k k} p_{n n}\right)^{-1}$. For a capacitor connected between two SSys as a capacitive $p i$ circuit, where $C_{i i}$ is in $S S y$ i and $C_{j j}$ in $S S y \mathrm{j}$, the convergence factor is given by $\gamma_{\ell}=\left(C_{i j} C_{j i}\right)\left[\left(C_{i i}+C_{i j}\right)\left(C_{j j}+C_{i j}\right)\right]^{-1}$. Finally, for a similar resistive coupling we have $\gamma_{\ell}=R_{i j}^{2} /\left[\left(R_{i i}+R_{i j}\right)\left(R_{j j}+R_{i j}\right)\right]$. It is clear that we can improve the accuracy of the criteria if more details of the connected circuits are taken into account. However, the majority of the entries in large PEEC circuit matrices are due to very weakly coupled delayed inductive and capacitive couplings.

We use the above partitioning process to determine which elements are in the same $S S y$. An example of a system after the partitioning process is given in Fig. 1 right. All the elements inside of SSys are strongly coupled and the dashed lines indicate a multitude of weak couplings. This shows where the weak couplings occur. From this, we also show that even inside the large $S S y 3$ we can update the weak couplings with each global WR iteration. Hence, we only have to solve a sparser SSy when we analyze SSy 3.

\section{RESULtS}

We use as an example a connector structure shown in Fig. 3. All measurements in the left figure are in $\mathrm{mm}$. To investigate different situations, we can add different layers to the connector. We assume that the connector connects to a set of contacts at a 90 degree angle. The contacts are assumed to be $0.4 \mathrm{~mm}$ thick with the conductivity of copper. We also assume that a $0.3 \mathrm{~mm}$ thick ground sheet is placed outside at a center-to-center distance of $2 \mathrm{~mm}$ from the connector wire. The size of the ground sheet is $29 \mathrm{~mm}$ by 17 $\mathrm{mm}$. Given these building blocks, we can construct an example with different connection situations to test the algorithm for different cases. As can be observed, the contacts of the connectors are labeled where we connect different resistive, capacitive and short loads to the connections indicated by the numbers. To simplify the example, all circuit elements and ground connections are assumed to be perpendicular to the planes and their 

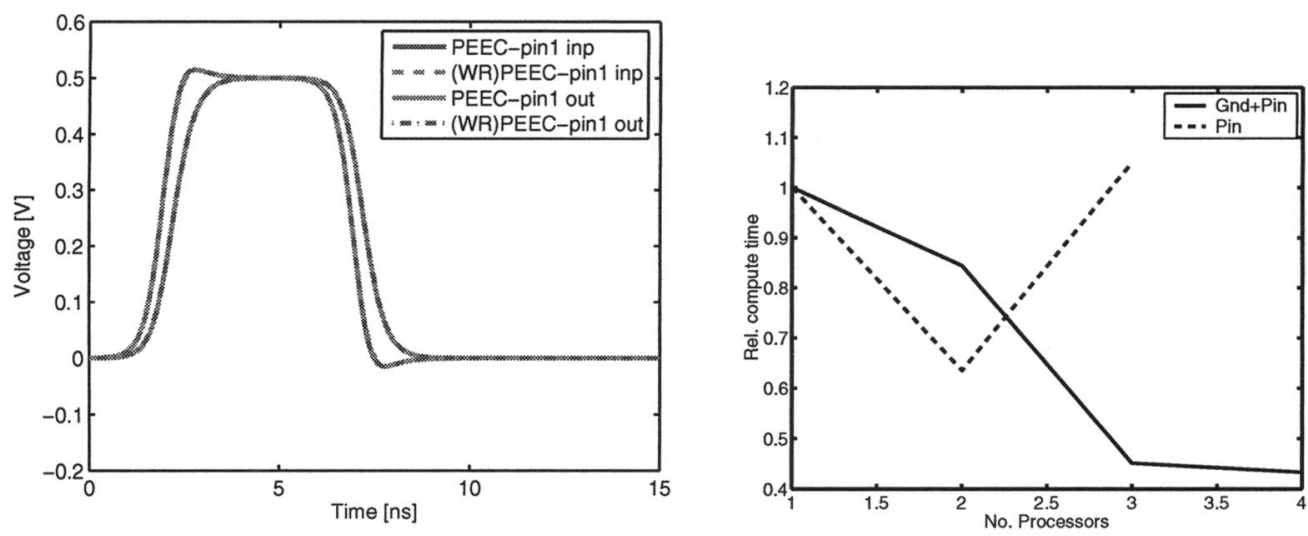

Fig. 4. Left: Waveform WR to flat validation. Right: Compute time vs processors for matrix problem

small connection impedance is ignored. Hence, the connection points are approriately chosen at the edge of the ground sheet which is lined up with the contacts. We chose the following variety of connections for the test problem at hand.

\begin{tabular}{|c|c|c||c|c|c||c|c|c|}
\hline Con. & Left(b) & Down(e) & Con. & Left(b) & Down(e) & Con. & Left(b) & Down(e) \\
\hline \hline 1 & $1 \mathrm{~V}+50 \mathrm{Ohms}$ & $50 \mathrm{Ohms}$ & 2 & Short & Open & 3 & $1 \mathrm{~V}+50 \mathrm{Ohms}$ & Open \\
4 & Open & Open & 5 & $1 \mathrm{~V}+100 \mathrm{Ohms}$ & $0.1 \mathrm{pF}$ & & & \\
\hline
\end{tabular}

The voltage source is assumed to be a $1 \mathrm{~V}$ step with a rise time of $0.05 \mathrm{~ns}$. In principle we could assign one processor to each of the partitioned pins with the exception of pin 2 which is shorted to the ground. Hence, the ground sheet and pin 2 are in one SSy. Our meshing resulted in $552 \mathrm{Lp}$-cells and $752 \mathrm{C}$-cells with a total of 200 nodes. However, the cells assigned to the ground plane and grounded pin are almost half of the total 264 Lp-cells and 304 C-cells and 80 nodes. Hence, it should be desirable to assign multiple processors to solve the ground plane problems by using the matrix solution. This is done with the Gaussian matrix solver to reduce the compute time for the larger ground sheet $S S y$ compute time. Figure 4 right shows how a number of processors assigned to the single pin is best at 2 while at least 3 processors should be assigned to the ground sheet and pin problem. The machine used is a Linux machine using MPI where the parallel matrix solver used is from ScaLaPack. Of course, this result is a strong function of the machine communication latency. We show that by using a parallel matrix solver we have some control over the execution time for each of the SSys. Hence, this simplifies the scheduling task for the different $S S y$ resulting in the balancing of the loads on the different processors. Figure 4 Left finally compares the input and output responses for pin 1 by comparing the WR response to the conventional Spice analysis waveforms.

\section{Conclusions}

This paper combines two parallel processing based speedup techniques for the solution of large EM problems using the PEEC approach. A conventional parallel Gaussian linear system solver is used for strongly coupled parts while the waveform relaxation method is used for the weak inductive and capacitive couplings. Hence, the approach uses both approaches where they perform the best.

\section{REFERENCES}

[1] A.E. Ruehli, G. Antonini, J. Esch, J. Ekman, A. Mayo, and A. Orlandi. Non-orthogonal PEEC formulation for time and frequency domain EM and circuit modeling. IEEE Transactions on Electromagnetic Compatibility, 45(2):167-176, May 2003.

[2] J. White and A. L. Sangiovanni-Vincentelli. Partitioning algorithms and parallel implementations of waveform relaxation algorithms for circuit simulation. In IEEE Proc. Int. Symp. on Circuits and Systems (ISCAS), pages 1069-1072, June 1985.

[3] A. E. Ruehli, Ed. Circuit analysis, simulation and design, Part 2. Elsevier Science Publishers B. V. (North-Holland), 1987.

[4] W. P. Pinello, A. E. Ruehli. Time domain solutions for coupled problems using PEEC models with waveform relaxation. In Proc. IEEE Antennas Prop. Society International Symp., volume 3, pages 2118-2121, Baltimore, MD, July 1996.

[5] J. Ekman and P. Anttu. Parallel implementation of the PEEC method. In Proc. of the IEEE Int. Symp. on Electromagnetic Compatibility, Honolulu, Hawaii, 2007.

[6] G. Antonini, A. E. Ruehli, and J. Ekman. Waveform relaxation for the parallel solution of large PEEC model problems. In Proc. of the IEEE Int. Symp. on Electromagnetic Compatibility, Honolulu,Hawaii, 2007.

[7] K. Burrage. Parallel and sequential methods for ordinary differential equations. Clarendon Press Oxford, New York, 1995.

[8] A. E. Ruehli and T. A. Johnson. Circuit analysis computing by waveform relaxation, volume 3 . Wiley Encyclopedia of Electrical Electronics Engineering, New York, 1999.

[9] N. J. Nakhla, A. E. Ruehli, M. S. Nakhla, and R. Achar. Simulation of coupled interconnects using waveform relaxation and transverse partitioning. IEEE Transactions on Advanced Packaging, 29(1):78-87, 2006.

[10] V. Jandhyala, S. Chakroborty, D. Gope, C. Yang, I. Choudhury, and G. Ouyang. Accelerated parallelized time and frequency domain simulation for complex high-speed microsystems. In Proc. IEEE Antennas Prop. Society International Symp., number 10.1109, pages $123-126$, September 2006. 